\title{
GESTIÓN DE TALENTO HUMANO PARA LA MEJORA LABORAL EN EL BANCO SOLIDARIO
}

\author{
Karla Verónica Castro Vivar* \\ https://orcid.org/0000-0003-4113-2715 \\ Kléber Antonio Luna Altamirano** \\ https://orcid.org/0000-0002-4030-8005 \\ Juan Carlos Erazo Álvarez ${ }^{* * *}$ \\ https://orcid.org/0000-0001-6480-2270
}

RECIBIDO:Septiembre 2019 / ACEPTADO: Noviembre 2019 / PUBLICADO: Enero 2020

Como citar:Castro Vivar, Karla; Luna Altamirano, Kléber; Erazo Álvarez, Juan. (2020). Gestión de Talento Humano para la mejora laboral en el Banco Solidario.Telos: revista de Estudios Interdisciplinarios en Ciencias Sociales, 22 (1), Venezuela. (Pp. 184-203).

DOI: www.doi.org/10.36390/telos221.13

\section{RESUMEN}

El Banco Solidario ubicado en la ciudad de Cuenca-Ecuador, es considerado una institución financiera solvente de servicio a la sociedad cuencana desde el año 2006 hasta la presente fecha; sin embargo, en dicha institución se evidencia la carencia de una comunicación adecuada entre los funcionarios. El objetivo es diseñar un Modelo de Gestión de Talento Humano, con el propósito de mejorar el desempeño laboral de los empleados dentro de esta institución financiera. Entre los principales autores que sostienen esta investigación, están Robbins y Coulter (2010), Chiavenato (2009), Restrepo y Ravagli (2009) y Tejada (2003), entre otros; es de orden descriptivo, con un enfoque cuantitativo que se apoya en el método deductivo. Se diseñaron encuestas dirigidas al personal de rangos jerárquicos con mayor conocimiento en esta área, quienes aportaron información relevante para el cumplimento del objetivo planteado. Los resultados arrojan excesivas horas de trabajo y baja remuneración salarial, además de la excesiva rotación de personal que no permite el compromiso laboral de los empleados del Banco Solidario. En función de ello, se propuso un modelo de gestión de talento humano para una correcta contratación del recurso humano, en función de un perfil establecido, de manera que se garantice su permanencia en la organización y por ende su estabilidad. Se tratará de reducir la rotación de personal ampliando el compromiso laboral. La institución financiera al contar con un talento humano comprometido con los ideales institucionales fidelizara a todos los trabajadores y con ello encaminarse al éxito empresarial.

\footnotetext{
"Universidad Católica de Cuenca, Posgradista Maestría en Administración de Empresas, Correo electrónico:kcastrov@psg.ucacue.edu.ec

***Profesor Investigador, Unidad Académica de Administración, Universidad Católica de Cuenca. Correo electrónico:klunaa@ucacue.edu.ec

***Universidad Católica de Cuenca, Subdirección de Posgrado, Cuenca, Ecuador, Correo electrónico:icerazo@ucacue.edu.ec
} 
Palabras clave: Desempeño laboral, modelo de gestión, talento humano, rotación de personal.

\title{
Human Talent Management for work improvement in Solidarity Bank
}

\begin{abstract}
The Solidarity Bank located in the city of Cuenca-Ecuador, is considered a solvent financial institution serving the Cuenca society from 2006 to the present date; However, the lack of adequate communication between officials is evidenced in that institution. The objective is to design a Human Talent Management Model, with the purpose of improving the work performance of employees within this financial institution. Among the main authors who support this research are Robbins and Coulter (2010), Chiavenato (2009), Restrepo and Ravagli (2009) and Tejada (2003), among others; It is descriptive, with a quantitative approach that relies on the deductive method. Surveys aimed at personnel of hierarchical ranks with greater knowledge in this area were designed, who provided relevant information to fulfill the objective. The results show excessive hours of work and low salary compensation, in addition to the excessive turnover that does not allow the labor commitment of the employees of Banco Solidario. Based on this, a human talent management model is proposed for the correct hiring of the human resource, based on an established profile, so as to guarantee its permanence in the organization and therefore its stability. It will try to reduce the turnover of personnel by expanding the labor commitment. The financial institution to have a human talent committed to institutional ideals will retain all workers and thereby move towards business success.
\end{abstract}

Keywords: Work performance, management model, human talent, staff turnover.

\section{Introducción}

El Banco Solidario fue creado en el año de 1996, en Latinoamérica fue el primer banco especializado en microcrédito con capital $100 \%$ privado y en Ecuador a dedicarse con exclusividad a esta forma de operación financiera, brindando créditos a personas de alta vulnerabilidad económica, el propósito es dar servicios a la microempresa, considerado como un segmento desatendido por la banca tradicional y de vital importancia para generar progreso (La República, 2013). Actualmente, el Banco Solidario, cuya actividad económica es netamente prestación de crédito tanto para pequeñas y medianas empresas, así también otorga créditos de consumo, en la actualidad la esta entidad financiera tiene varias sucursales a nivel nacional, una de ellas se localiza en la ciudad de Cuenca en la calle Benigno Malo y Gran Colombia, siendo la única sucursal dentro de esta urbe.

El problema reflejado se desprende de una mínima existencia de comunicación dentro de los departamentos y entre funcionarios, lo más relevante es la excesiva rotación de personal, la misma que se le observa desde dos enfoques, la primera es por disposición de la gerencia, y la segunda es por renuncia voluntaria, generando inestabilidad y un ambiente laboral hostil, y por parte de los clientes un cierto grado de insatisfacción y desconfianza, Robbins (1998, p.343) sostiene:

La rotación de personal es el retiro voluntario e involuntario permanente de una organización. Puede ser un problema, debido al aumento de los costos de reclutamiento, de selección, de capacitación y de los trastornos laborales. No se 
puede eliminar, pero se puede minimizar, sobre todo entre los empleados con un alto nivel de desempeño y de los difíciles de reemplazar.

Robbins y Coulter (2010, p.214) afirman:

Que para aumentar la satisfacción del empleado y reducir la rotación de personal, los gerentes deben considerar el uso de una sinopsis de trabajo realista (STR), la cual incluye tanto la información positiva como la negativa del puesto y de la compañía.

Los mismos autores Robbins y Coulter (2010, p.236), con relación a la comunicación, aseveran:

La comunicación abierta y bilateral importante para manejar un equipo diverso. Los problemas de diversidad pueden intensificarse si las personas temen o no tienen confianza de expresar abiertamente problemas que les conciernen.

El objetivo del estudio, fue diseñar un Modelo de Gestión de Talento Humano, con la finalidad de mejorar el desempeño laboral de los empleados dentro de esta institución financiera, se puede decir que el desempeño de un funcionario está representado por distintas características que lo posee, como habilidades experiencias, destrezas, actitudes, aptitudes (eficiencia y eficacia) y valores personales que le apoyan a lograr las metas esperadas, acorde a las exigencias de la empresa, Chiavenato (2009), explica que a la gestión del talento humano se le considera como un conjunto de prácticas y políticas necesarias para guiar las funciones de los cargos gerenciales relacionados con el recurso humano, entre ellos, la selección, capacitación, condecoraciones y evaluación de desempeño. La participación en el trabajo es el grado en que un empleado se identifica con su trabajo, participa activamente en él y considera que su desempeño laboral es importante para su propia valía (Robbins y Coulter, 2010, p.307). Este representa un compromiso para mejorar el conocimiento, capacidades y habilidades del recurso humano dentro de una organización. La presente investigación es de tipo descriptivo, el cual detalla con exactitud el objeto de estudio, no solo se recolecta datos sino se estudia y se analiza los resultados más relevantes los cuales constituyen aporte al conocimiento, Cazau (2006, p.27) sostiene:

En un estudio descriptivo se seleccionan una serie de cuestiones, conceptos o variables y se mide cada una de ellas independientemente de las otras, con el fin, precisamente, de describirlas. Estos estudios buscan especificar las propiedades importantes de personas, grupos, comunidades o cualquier otro fenómeno.

El presente estudio, tuvo su enfoque desde lo cualitativo, en donde se describe las cualidades de un fenómeno, buscando concepciones que pueda contener una parte de la realidad. Aunado a esto, se planteó el enfoque cuantitativo, el cual utiliza la recolección de datos para probar hipótesis con base en la medición numérica y el análisis estadístico, con el fin establecer pautas de comportamiento y probar teorías (Hernández, Fernández y Baptista, 2014, p.4).

El método que se aplicó fue el deductivo, ya que este se refiere a una forma del razonamiento o pensamiento, parte de lo general para llegar a algo específico. El método deductivo, es una forma de razonamiento que parte de una verdad universal para obtener conclusiones particulares (Maya, 2014, p.14). La aplicación este modelo de gestión de talento humano, logrará aposteriorí solucionar los problemas inherentes a la insatisfacción del personal que labora dentro del Banco Solidario Sucursal-Cuenca.

El artículo se estructura, indicando en primera instancia la revisión del estado del arte, en el 
cual se soporta teóricamente la investigación, partiendo de la revisión de investigaciones sobre el tema en mención. Como parte de la metodología se detalla la construcción del modelo de gestión de talento humano. Posteriormente, se plantean los resultados orientados a la mejora de la gestión institucional, propiciando un ambiente laboral apropiado para el desarrollo de las funciones que desempeñan cada uno de los funcionarios, como conclusión se logrará obtener un recurso humano eficiente y eficaz, con ello se generará una mayor producción logrando resultados óptimos.

\section{Estado del arte}

El diseñar un modelo de Gestión de Talento Humano para el Banco Solidario de la ciudad de Cuenca-Ecuador, es de suma importancia ya que este permitirá conocer la satisfacción y motivación del personal que labora en esta institución financiera, por ello algunos autores contribuyen con sus investigaciones relacionados al tema de estudio, entre ellos: Osorio, Navarrete y Martínez (2009), proponen un modelo de gestión, con el propósito de estructurar un Banco de talentos empresariales con un alto componente de ciencia, tecnología e Innovación para que aporte al desarrollo económico, social y competitivo del municipio de Pereira-Colombia. Tejada (2003), realiza un análisis de los tres modelos de gestión que en la actualidad tienen un significado histórico y contextual dentro de las empresas: la gestión del talento humano, la gestión del conocimiento y la gestión por competencias, cuyo propósito es indicar que estos modelos de gestión están claramente relacionados e integrados entre sí y que se deben ser estudiados como una unidad.

Argüelles (2011), presenta el diseño de un modelo para la generación de competencias genéricas a partir del e-Learning, basado en aprendizaje autónomo, que considera no sólo las necesidades de formación y capacitación dentro de la organización, en términos coyunturales, sino, además, a la urgencia de desarrollar un talento humano, capaz de ponerse a tono, por sí mismo, con las exigencias del entorno, en el marco de la empresa. Restrepo y Ravagli (2009) exponen un modelo de administración de Talento Humano para una empresa en el área de producción como parte del direccionamiento estratégico, con ello en lo posterior diseñar los procesos de la cadena de valor, con la finalidad de identificar como estos impactan en el cumplimiento de la misión, visión, políticas y factores de éxito.

Por su parte, los autores Jara, Asmat, Alberca y Medina (2018), presentan su investigación con la finalidad de determinar la incidencia de la gestión del talento humano en el mejoramiento de la gestión pública y el desempeño laboral de los trabajadores de la Administración central del Ministerio de Salud del Perú. Gallardo, González, Martínez y Pardo (2012), explican su estudio desde dos enfoques, el primero realiza una revisión de la literatura especializada con respecto a la conceptualización de talento humano y la función del departamento de recursos humanos dentro de la gestión de talento humano, el segundo enfoque se refiere a la aplicación del método Delphi para las empresas españolas con el propósito de obtener la percepción de los expertos sobre el rol del departamento de talento humano con relación a la crisis en la gestión de talento.

Rodríguez y Rosenstiehl (2018), determinan la probabilidad de que el bajo desempeño en la gestión de operaciones logística en América Latina se presente por la carencia de recurso humano especializado en actividades logística, la información obtenida lo analizaron a través de un análisis factorial confirmatorio y Regresión Logística, la investigación parte de una 
hipótesis la cual es parte de la teoría de eficiencia, técnica a partir de los trabajos de Farrel (1957). Restrepo, Ladino y Orozco (2008), diseñan de un modelo de Reclutamiento y Selección de Talento Humano por competencias para los puestos de los niveles Directivo de una organización, con ello permita elegir nuevos funcionarios eficientes que contribuyan al progreso empresarial, toando en consideración que las personas con sus capacidades y su motivación son las que verdaderamente crean la ventaja competitiva y establecen la diferencia en las empresas de éxito.

Otros autores, como Majad (2016); Suárez (2011); y Mejía, Bravo y Montoya (2013), estudian la gestión del talento humano en el ámbito empresarial en diferentes tipos de organizaciones, preciando dilemas en la postmodernidad y otros elementos de interés sobre esta temática. Asimismo, Falconi, Luna, Sarmiento y Andrade (2019), precisan estudios sobre gestión administrativa y sus relaciones con los procesos humanos. Los autores aportan a un correcto desarrollo de la construcción de un modelo de gestión de talento humano, ya que tiene una relación directa con la planificación estratégica de la institución bancaria, con ello se podría mejorar el nexo y crear interdependencia, así se propenderá a optimizar la gestión empresarial dando lugar a mejoras continuas con la finalidad de aportar al crecimiento de la organización.

\section{Metodología}

La presente investigación es de tipo descriptivo con enfoque cuantitativo por lo que se aplica el método deductivo para el diseño de un modelo de gestión de talento humano, la información es suministrada a través de una encuesta dirigida a 25 funcionarios del Banco Solidario de la Sucursal de Cuenca, quienes conforman el universo total de trabajadores, con el propósito de dar mayor veracidad a esta información. A continuación, se detallan los cargos del personal a quienes estará dirigida la encuesta:

Tabla 1. Segmentación de empleados del Banco Solidario

Personal total Numero

Gerencia comercial

Gerencia microcrédito

Jefe operativo 
Oficial de inversiones

Oficial de negocios

Asesor microcréditos

Asistente de microcrédito

Plataforma comercial

Perito evaluador

Cajero

Señoras limpiezas

Guardias de seguridad

Total, de empleados

Fuente: Elaboración propia

\section{Resultados}

Como se puede evidenciar en la tabla 1, la población de los trabajadores del Banco Solidario de la sucursal de la ciudad de Cuenca, son de 25 empleados, a los cuales se realizó el estudio mediante una encuesta, las edades de estos funcionarios fluctúan entre 23 a 50 años, son personas que cuentan con un título de tercer nivel de estudios, tienen experiencia dentro de la banca y son conocedores de la problemática que atraviesa la entidad financiera. La información más relevante de las encuestas se presenta a continuación: 


\section{Gráfico 1. Capacitación e inducción}

¿Cómo fue la capacitación e inducción que recibió al puesto de trabajo para el desempeño de sus funciones?

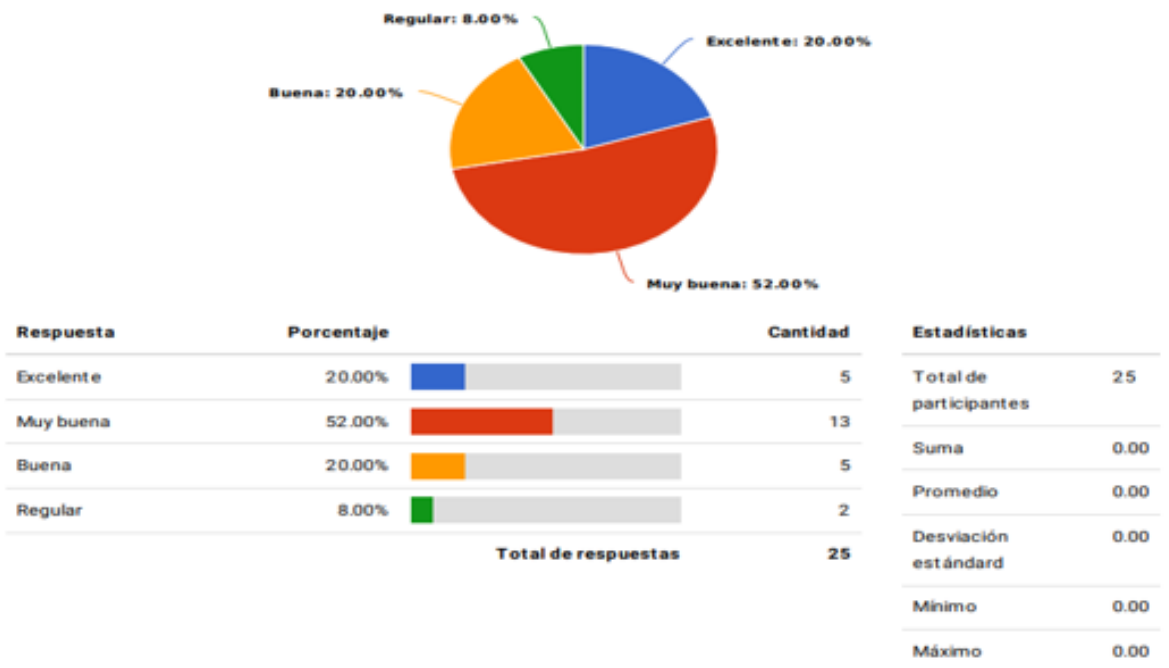

Fuente: Elaboración propia

Si se suma la respuesta de muy buena y buena, se tiene un porcentaje del $62 \%$, significa que el personal de acuerdo con su puesto de trabajo no fue capacitado de manera adecuada. La capacitación permite que los funcionarios incrementen sus conocimientos, destrezas y habilidades, con el propósito de generar mayores beneficios a la institución. Por ello López (2005) explica que una de las funciones de la empresa, es capacitar a su personal de manera obligatoria y su administración debe llevarse de forma adecuada, esta capacitación debe realizarse de acuerdo a las necesidades de cada departamento, con ellos los trabajadores cumplirán sus actividades de manera eficiente. 
Gráfico 2. Reclutamiento del personal

\section{¿Cómo considera que es el reclutamiento del personal de la empresa?}

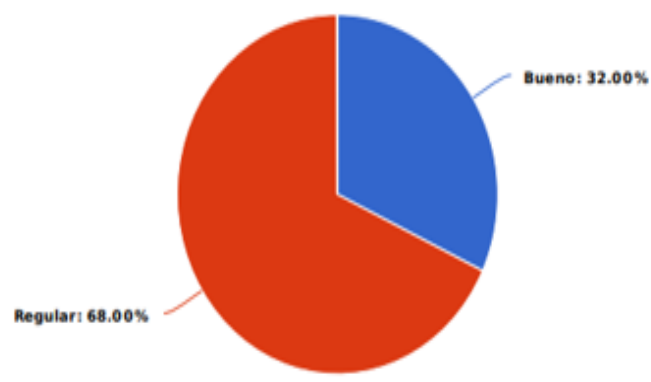

\begin{tabular}{|c|c|c|c|c|c|}
\hline Respuesta & Porcentaje & & Cantidad & Estadisticas & \\
\hline Bueno & $32.00 \%$ & & 8 & Total de & 25 \\
\hline Regular & $68.00 \%$ & & 17 & Icipantes & \\
\hline \multirow[t]{6}{*}{ Malo } & $0.00 \%$ & & 0 & Suma & 0.00 \\
\hline & & Total de respuestas & 25 & Promedio & 0.00 \\
\hline & & & & Desviación & 0.00 \\
\hline & & & & estándard & \\
\hline & & & & Minimo & 0.00 \\
\hline & & & & Máximo & 0.00 \\
\hline
\end{tabular}

Fuente: Elaboración propia

Respecto al reclutamiento del personal un $68 \%$ de los empleados comentan que el procedimiento de reclutamiento fue regular, con esto se concluye que el reclutamiento no es adecuado no cumplió las expectativas de cada uno de los empleados. El reclutamiento de personal, consiste en seleccionar candidatos apropiados para un determinado cargo, ya que este debe cumplir de manera eficiente el trabajo asignado. Flores (2016, p.81) afirma:

El proceso de reclutamiento y selección de personal es definido como acciones planeadas y emprendidas hacia el descubrimiento y medición de las particularidades personales de cada postulante, con la finalidad de establecer 
cuáles son los más idóneos para ejercer determinados quehaceres en la organización.

Gráfico 3. Equipos de oficina

\section{¿Cuenta usted con los equipos y/o herramientas necesarias para realizar sus funciones?}

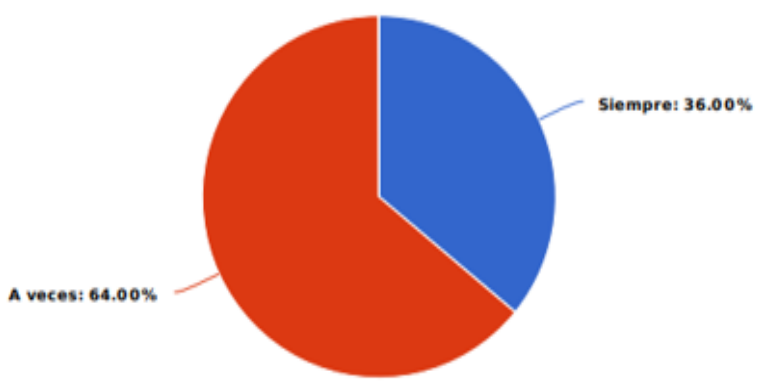

\begin{tabular}{|c|c|c|c|c|c|}
\hline Respuesta & Porcentaje & & Cantidad & Estadisticas & \\
\hline Siempre & $36.00 \%$ & & 9 & Total de & 25 \\
\hline A veces & $64.00 \%$ & & 16 & ive & \\
\hline \multirow[t]{6}{*}{ Nunca } & $0.00 \%$ & & 0 & Suma & 0.00 \\
\hline & & al derespuestas & 25 & Promedio & 0.00 \\
\hline & & & & Desviación & 0.00 \\
\hline & & & & estándard & \\
\hline & & & & Minimo & 0.00 \\
\hline & & & & Máximo & 0.00 \\
\hline
\end{tabular}

Fuente: Elaboración propia

Con respecto a dotación de equipos de oficina, el $64 \%$ de los funcionarios expresan que pocas veces tienen lo necesario para desarrollar sus funciones de manera adecuada. Todo funcionario que labora en una oficina debe tener un ambiente agradable de trabajo, este debe contar con buenos equipos y mobiliarios para un mejor desempeño. La maquinaria y equipos de oficina comprenden todos los artículos necesarios para desarrollar con eficiencia los procesos productivos o prestación de servicios, su 
selección debe realizarse de acuerdo a las características de cada puesto de trabajo (Córdoba, 2011).

Gráfico 4. Competencias establecidas

¿En su puesto o área de trabajo actual, labora usted por competencias establecidas entre directivos y personal para alcanzar objetivos?

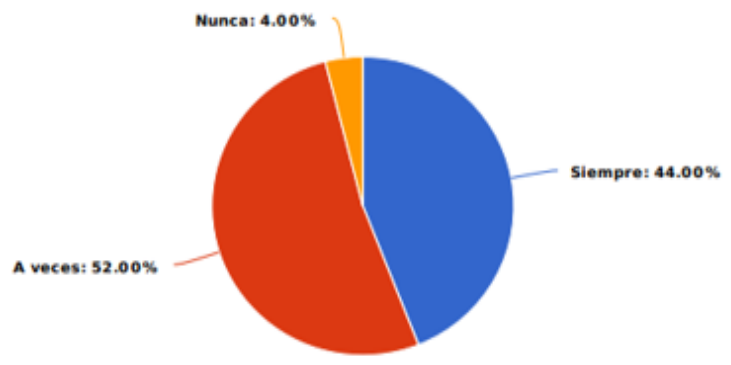

\begin{tabular}{|c|c|c|c|c|c|}
\hline Respuesta & Porcentaje & & Cantidad & Estadisticas & \\
\hline Siempre & $44.00 \%$ & & 11 & Total de & 25 \\
\hline A veces & $52.00 \%$ & & 13 & s & \\
\hline \multirow[t]{6}{*}{ Nunca } & $4.00 \%$ & & 1 & Suma & 0.00 \\
\hline & & & & Promedio & 0.00 \\
\hline & & & & Desviación & 0.00 \\
\hline & & & & est ándard & \\
\hline & & & & Minimo & 0.00 \\
\hline & & & & Máximo & 0.00 \\
\hline
\end{tabular}

Fuente: Elaboración propia

En cuanto a las competencias de cada área de trabajo el colaborador establece que en un $52 \%$ a veces labora por objetivos establecidos entre directivo y personal, dando a conocer un porcentaje de insatisfacción respecto al correcto funcionamiento de competencias. El trabajo bajo competencias es un factor clave, ya a través de este cada funcionario puede ir estableciendo un determinado dominio sobre estas. López y Chaparro (2006, p.264) afirman:

Las competencias laborales se perciben como el punto de encuentro entre los sectores educativo y productivo, por cuanto muestran qué se debe formar en los trabajadores y los desempeños que éstos deben alcanzar en el espacio laboral. El surgimiento de la gestión por competencia laboral en la empresa, en 
parte obedece a la necesidad de acortar la distancia entre esfuerzo de formación y resultado efectivo.

Gráfico 5. Capacitación al personal

¿Las capacitaciones que recibe, siempre son acordes a su área de trabajo y funciones que desempeña?

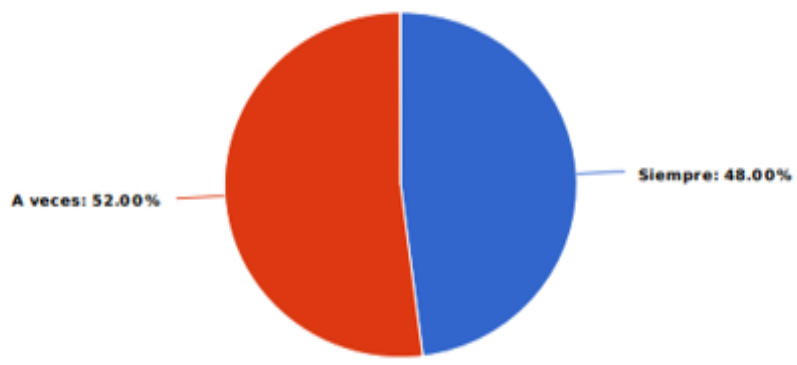

\begin{tabular}{|c|c|c|c|c|c|}
\hline Respuesta & Porcentaje & & Cantidad & Estadisticas & \\
\hline Siempre & $48.00 \%$ & & 12 & Total de & 25 \\
\hline A veces & $52.00 \%$ & & 13 & cipantes & \\
\hline \multirow{6}{*}{ Nunca } & $0.00 \%$ & & & Suma & 0.00 \\
\hline & & & & Promedio & 0.00 \\
\hline & & & & Desviación & 0.00 \\
\hline & & & & est ándard & \\
\hline & & & & Minimo & 0.00 \\
\hline & & & & Máximo & 0.00 \\
\hline
\end{tabular}

Fuente: Elaboración propia

Se puede visualizar un porcentaje del $52 \%$ los empleados, comentan que las capacitaciones no son acordes a su puesto de trabajo y las funciones a ellos encomendadas. Por ello la importancia de capacitar a los funcionarios con la finalidad de generar nuevos conocimientos, experiencias y capacidades, y con ello lograr un desempeño selecto. Sapién, Piñón y Gutiérrez (2014, p.124) sostienen:

La capacitación y el adiestramiento son formas de educación. Por medio de ellas, se adquieren conocimientos, habilidades y destrezas que son necesarios 
para lograr la eficacia y la excelencia en la realización de tareas, funciones y responsabilidades.

Gráfico 6. Evaluación al personal

¿Cumu colaborador del banco, es usted evaluado cada cierto período de tiempo, respecto a funciones y actividades que le asignaron?

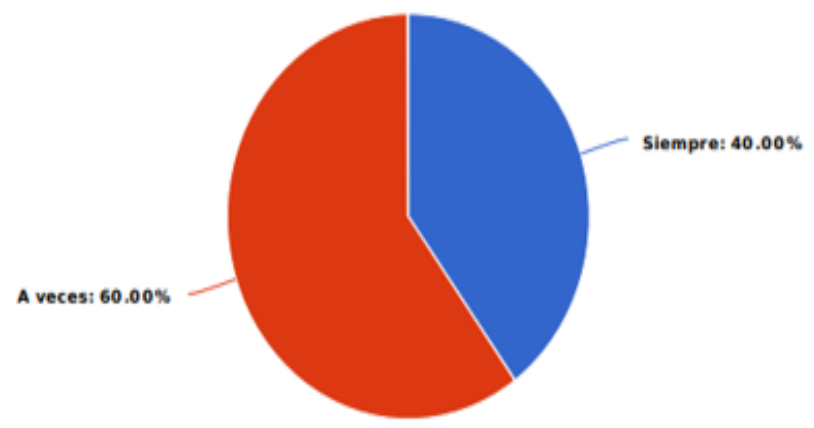

\begin{tabular}{|c|c|c|c|c|c|}
\hline Respuesta & Porcentaje & & Cantidad & Estadisticas & \\
\hline Siempre & $40.00 \%$ & & 10 & Total de & 25 \\
\hline Aveces & $60.00 \%$ & & 15 & articipantes & \\
\hline \multirow[t]{6}{*}{ Nunca } & $0.00 \%$ & & 0 & Suma & 0.00 \\
\hline & & & & Promedio & 0.00 \\
\hline & & & & Desviación & 0.00 \\
\hline & & & & estándard & \\
\hline & & & & Minimo & 0.00 \\
\hline & & & & Máximo & 0.00 \\
\hline
\end{tabular}

Fuente: Elaboración propia

La evaluación de funciones al personal, no es acogido de buena manera por un $60 \%$ de consultados, mencionan que pocas veces el área pertinente es evaluada conjuntamente con las diferentes actividades desarrolladas por cada colaborador. La evaluación al desempeño de un trabajador es importante, ya que este permite medir el rendimiento en las funciones encomendadas a cada funcionario, con ello establecer estrategias para el mejoramiento del desempeño laboral. La evaluación del desempeño laboral en una 
organización, tiene la finalidad de valorar de forma sistemática y objetiva, el rendimiento o desempeño de los empleados o funcionarios (Salgado y Cabal, 2011)

Gráfico 7. Rotación de personal

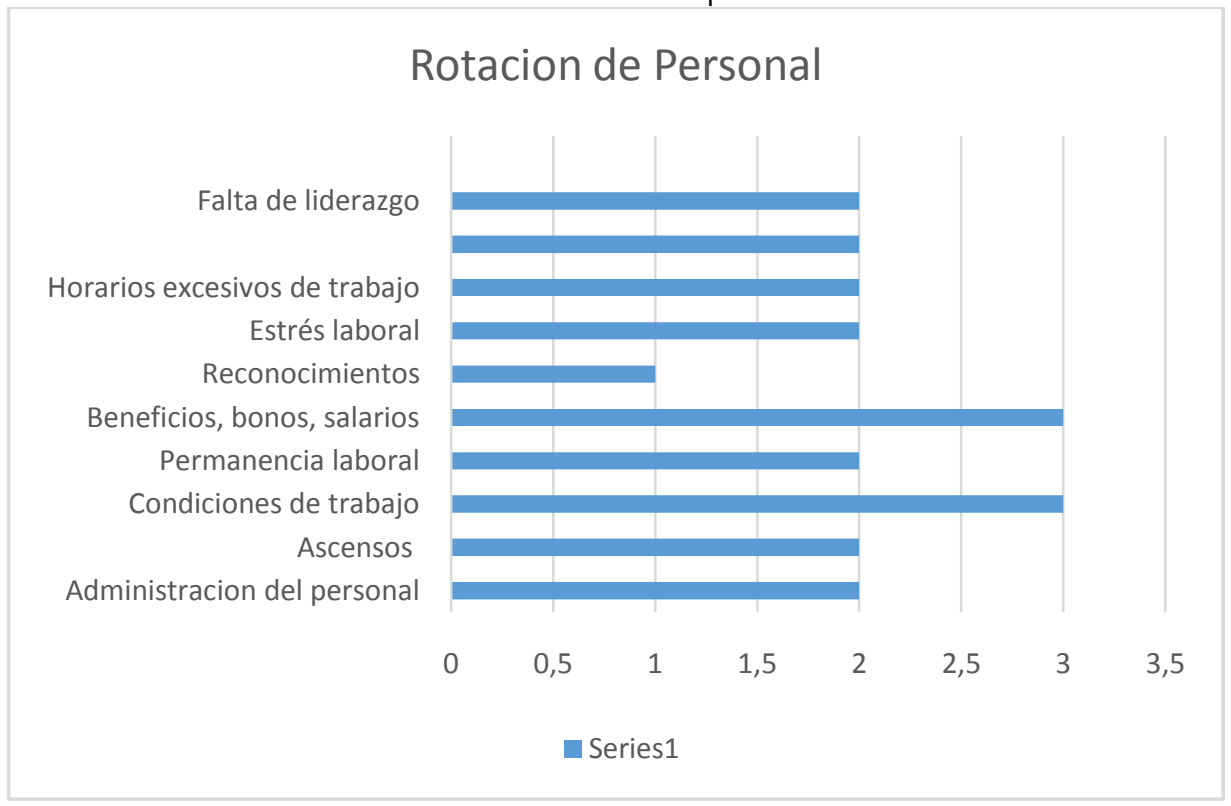

Fuente: Elaboración propia

Se puede observar que las condiciones de trabajo, beneficios, bonos y salarios son de mayor relevancia, por este motivo existe la gran rotación de personal dentro de la entidad financiera. Un alto nivel de rotación de personal dentro de una empresa, origina un efecto negativo, ya que el personal deberá estar en constantes capacitación con el propósito de mejorar su desempeño, en otros casos representa frustración y desmotivación en el puesto de trabajo. Chiavenato (1999, p.188) asevera:

El término de rotación de personal se utiliza para definir la fluctuación de personal entre una organización y su ambiente; esto significa que el intercambio de personas entre la organización y el ambiente se define por el volumen de personas que ingresan en la organización y el de las que salen de ella.

La información que se presenta, es significativa para el diseño del modelo de gestión de talento humano, para ello se hace necesario explicar la razón de rotación del personal, la 
misma se estructuró en base a los datos recabados a través de las encuestas, lo indicado se detalla en el siguiente gráfico.

Gráfico 8. Razón de rotación de personal

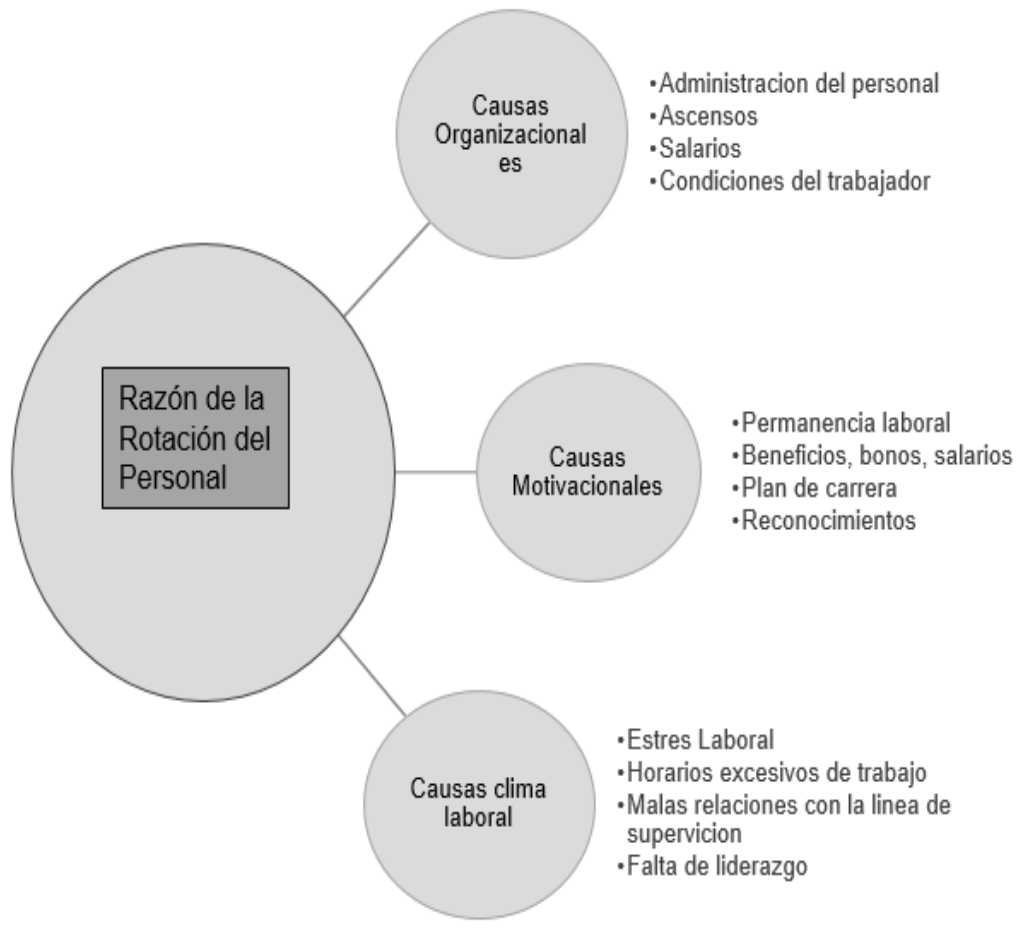

Fuente: Elaboración Propia

En el Gráfico 8, se puede observar las razones de la excesiva rotación de personal dentro del Banco Solidario, adicional a esto se suman los costos que representan a la entidad financiera tales como costo de reclutamiento, costo de selección, costo de integración, y costo de capacitación, entre otros.

Para el diseño del modelo de gestión de talento humano, y derivado del trabajo de campo realizado en la organización estudiada, se estructura la matriz FODA, en la cual se precisan fortalezas, oportunidades, debilidades y amenazas de la institución financiera. Es de destacar que la información para la estructura de estas matrices, se 
obtuvo de la misma encuesta realizada a los 25 funcionarios de la institución. A continuación, en la tabla 2 , se indican factores internos (fortalezas y debilidades) encontradas

Tabla 2. Matriz FODA Factores Internos

- Mejora continua de competencias técnica

- Políticas y procedimientos establecidos por recursos humanos

- Apertura a un cambio

- Ubicación físico y virtual

- Amplios conocimientos en la selección y contracción del personal

- Capacidad de innovación
- Personal no idóneo

- No existe un sistema de gestión de competencia

- Se vincula al personal, mas no se capacita

- Deficiente presupuesto asignado para la capacitación

- Reducida integración del personal

- Planes y programas insuficiente

Fuente: Elaboración Propia

Una vez realizado la matriz FODA, en la variable tanto internas como externas que son las que influyen de manera directa en el desempeño de los distintos trabajadores, se debe evaluar paso a paso la situación en la que se encuentra la entidad internamente, además se determina las fortalezas y debilidades, más relevantes de la institución financiera, aquí se visualiza puntualmente la situación actual de la entidad en estudio, cada una de la variables permitirá tomar decisiones estratégicas será el nexo que permita ver la realidad del ambiente interno para un correcto desempeño del plan de mejora del recurso humano dentro de la institución, lo principal observado en el FODA es la necesidad de cambios en las fortalezas y debilidades ya que en estas variables se puede tomar decisiones de permuta con la finalidad de crear un ambiente laboral adecuado, evitando la rotación de personal, además de un correcto reclutamiento de postulantes para los indistintas vacantes ofertadas.

Posteriormente, al desplegar los factores externos (oportunidades y amenaza), se tienen tabla 3: 
Tabla 3. Matriz FODA factores externos

\begin{tabular}{|c|c|}
\hline Oportunidades & Amenazas \\
\hline $\begin{array}{l}\text { - } \quad \text { Competencia laboral } \\
\text { - } \\
\text { Implementación de nuevos } \\
\text { sistemas y procesos } \\
\text { Capacitar al personal que } \\
\text { labora en la empresa } \\
\text { - } \text { Actualización de las } \\
\text { políticas de recursos } \\
\text { humanos dentro de la } \\
\text { organización } \\
\text { Condiciones de trabajo } \\
\text { para el personal } \\
\text { Alianzas estratégicas }\end{array}$ & $\begin{array}{ll}\text { - } & \text { Baja competencia laboral } \\
\text { - } & \text { Reducido personal } \\
\text { capacitado } \\
\text { - } \\
\text { Información de la empresa } \\
\text { a la competencia } \\
\text { - } \quad \text { Leyes laborales } \\
\text { - Inestabilidad para los } \\
\text { empleados }\end{array}$ \\
\hline
\end{tabular}

Fuente: Elaboración Propia

La matriz FODA permite establecer una correlación, así se puede visualizar la actual situación de la institución financiera para tener un correcto diagnóstico para la toma de decisiones adecuada así en la investigación permite definir y formular un contexto que permita caracterizar la problemática, definición y formulación que explique los lineamientos a seguir.

Se presenta el Modelo de Gestión de Talento Humano, en base al análisis de la información más relevante obtenida a través de las encuestas, la matriz FODA, fue importante para la estructura de este modelo, con ello se pretende generar cambios a nivel de talento humano del Banco Solidario de la ciudad de Cuenca-Ecuador.

Este diseño se indica a continuación (tabla 4): 
Tabla 4. Modelo de Gestión de Talento Humano

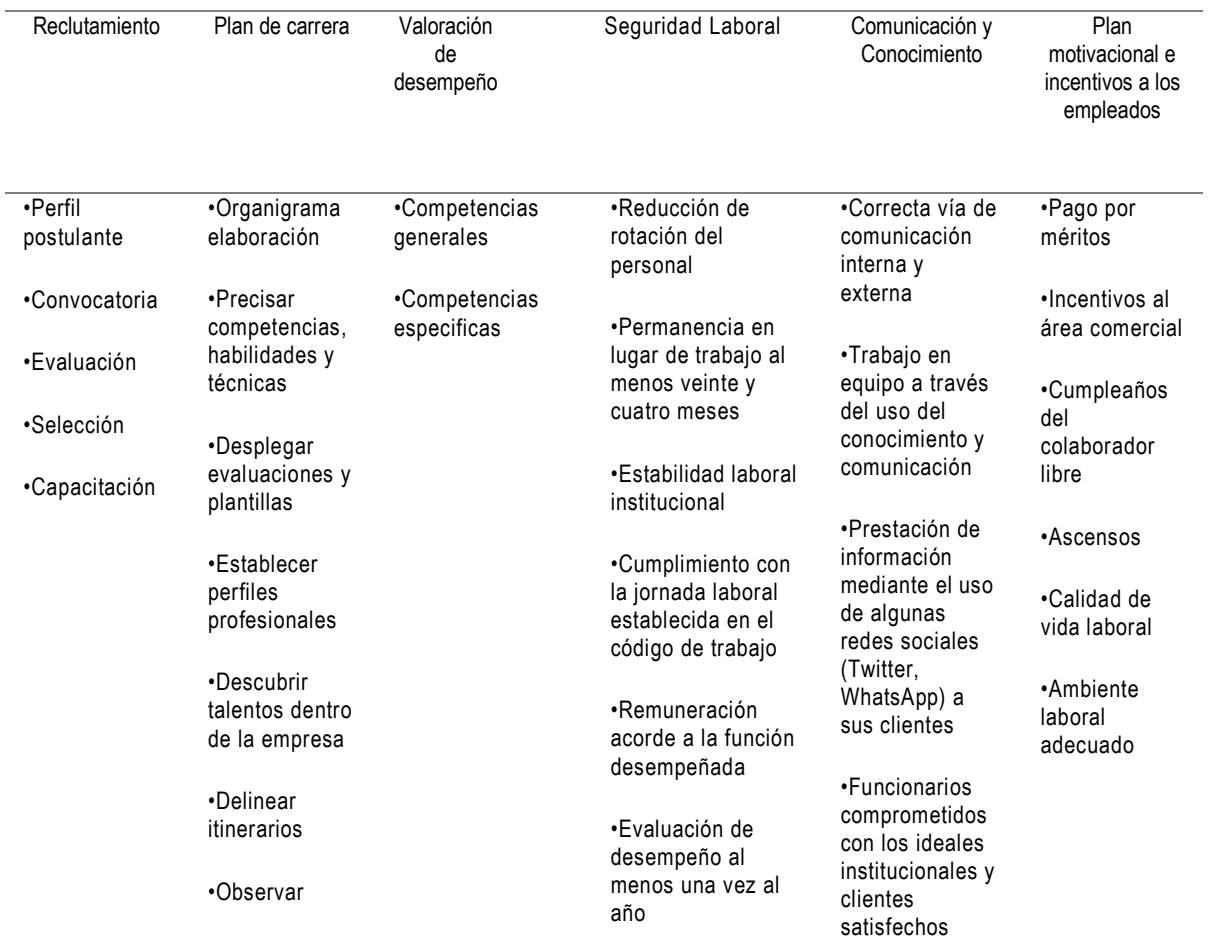

\section{Fuente: Elaboración propia}

En la tabla 4, se observa los cambios pertinentes que debe realizar la institución financiera. Este modelo de gestión de talento humano incorpora como elementos diferenciadores un ambiente de Seguridad Laboral y una correcta Comunicación a través del Conocimiento, con la finalidad de reducir la rotación de personal, la estabilidad tanto emocional como física de los empleados, la institución evitara gastos en el reclutamiento constante del personal generando un mejor ambiente laboral, con ello los empleados se sentirán parte de la empresa, se diseña este modelo con base a la información obtenida de las encuestas, como se mencionó anteriormente este instrumento se confecciono con alrededor de treinta preguntas, de ellas las más importantes se demuestra en este estudio. Con la aplicación de este modelo, la entidad financiera, mejoraría su gestión empresarial a través de: 
- Una correcta organización empresarial permitirá a la entidad financiera aplicar los recursos de manera apropiada y productiva.

- Los empleados que laboran dentro de la institución financiera tendrán un mayor compromiso con la misma, sin embargo, poner en práctica un incentivo adicional a su salario permitirá una satisfacción extra por el trabajo que desempeñan.

- La entidad camina siempre con el apoyo de los funcionarios y viceversa, con una misma finalidad de alcanzar objetivos.

- Contratación correcta del recurso humano, con el propósito de crear estabilidad laboral para cada uno de los empleados.

- Disminución en la rotación de personal, con ello se ampliará el compromiso laboral con el Banco.

- Canal de comunicación adecuado tanto interna como externa, a través del conocimiento para que cada funcionario de a conocer sus inquietudes a ser resueltas.

- Las horas de trabajo será la establecida por la ley, en caso de existir horas extras estas serán apegadas estrictamente a lo establecido en el código de trabajo ecuatoriano.

- Capacitación permanente a través de charlas y cursos motivacionales, con ello se tendrán funcionarios eficientes y dispuestos a trabajar con el objetivo institucional.

\section{Conclusiones}

El diseñar un Modelo de Gestión de Talento Humano, para el Banco Solidario de la ciudad de Cuenca-Ecuador, tiene el propósito de generar valor y capacidad de respuesta a las necesidades exigentes que requiere la entidad financiera, sus clientes y la sociedad en general. La propuesta presentada en este estudio da cumplimiento al objetivo planteado, y se orienta a gestionar de una manera más apropiada el recurso humano, mediante el diseño de estrategias para cada componente que conforma el modelo, con ello la institución financiera podrá optimizar el desempeño laboral.

Este representa una alternativa para el desarrollo del talento humano, que posibilita no solo el mejoramiento de la gestión institucional generando un ambiente laboral adecuado, reducción de rotación de los funcionarios dentro de las áreas de trabajo, remuneración justa con un horario de trabajo acorde a lo que estable el código de trabajo ecuatoriano, sino que, además, permite a la entidad financiera la optimización de los recursos mediante la eficiencia y eficacia del capital humano, incrementando la productividad y logrando que sus resultados sean mucho más óptimos.

El modelo de gestión de talento humano que se propuso, se enmarca en las necesidades institucionales, mediante competencias que permitan potenciar su recurso humano, convirtiéndose en capital organizacional, este permitirá generar ventajas competitivas para la misma, con ello se espera optimizar recursos económicos y financieros para poder llegar al éxito empresarial.

\section{Referencias bibliograficas}

Argüelles, Denise (2011). Un modelo para la formación del talento humano en la organización en el siglo XXI. Revista Escuela de Administración de Negocios, núm. 71, (Pp. 
148-161). Extraído de http://www.redalyc.org/articulo.oa?id=20623157010, consulta: 20/07/19

Cazau, Pablo. (2006). Introducción a la investigación en ciencias sociales. (tercera edición). Buenos Aires. Extraído de http://alcazaba.unex.es/asg/400758/MATERIALES/INTRODUCCI\%C3\%93N\%20A\%2 OLA\%20INVESTIGACI\%C3\%93N\%20EN\%20CC.SS..pdf, consulta: 16/09/19

Chiavenato, Idalberto. (1999). Administración de Recursos Humanos(quinta edición). Mc Graw-Hill Interamericana. México.

Chiavenato, Idalberto. (2009). Gestión del talento humano (tercera edición). McGraw Hill. México.

Córdoba, Marcial. (2011). Formulación y Evaluación de Proyectos (segunda edición). Ecoe Ediciones. Bogotá, Colombia.

Falconi, Juan., Luna, Kléber., Sarmiento, William., y Andrade, Celio. (2019). Gestión administrativa: Estudio desde la administración de los procesos en una empresa de motocicletas y ensamblajes. Revista Visionario Digital, Vol. 3, No. 2, (Pp. 197-212). Extraído de https://doi.org/10.33262/visionariodigital.v3i2.406, consulta: 18/09/19

Flores, José. (2016). Estrategias para mejorar el proceso de reclutamiento y selección de personal en la Dirección de teleinformática de la gobernación del estado Mérida. Sapienza Organizacional, Vol. 3, No. 5, (Pp. 79-102). Extraído de http://www.redalyc.org/articulo.oa?id=553057362005. Consulta: 12/10/19.

Gallardo, Eva., González, Tomás., Martínez, Clara., y Pardo, Manuela. (2012). Gestión del talento en la empresa española. Rol del departamento de recursos humanos. Revista Venezolana de Gerencia, Vol. 17, No. 58, (Pp. 232-252). Extraído de: http://www.redalyc.org/articulo.oa?id=29023348003, consultada:02/07/19

Hernández, Roberto., Fernández, Carlos., y Baptista, María. (2014). Metodología de la Investigación (sexta edición). McGraw-Hill. Educación. México.

Jara, Antonio., Asmat, Nicanor., Alberca, Nancy., y Medina, Jessica. (2018). Gestión del talento humano como factor de mejoramiento de la gestión pública y desempeño laboral. Revista Venezolana de Gerencia, Vol. 23, No. 83. Extraído de: http://www.redalyc.org/articulo.oa?id=29058775014, consulta: 19/09/19

La República (2013). Banco Solidario y Unibanco se integran, Extraído de https://www.larepublica.ec/blog/economia/2013/06/10/banco-solidario-y-unibanco-seintegran/, consulta 18/08/19

López, Emilia., y Chaparro, Melba. (2006). Competencias laborales del trabajador vistas desde el mercado laboral (parte A). Tabula Rasa, No. 5, (Pp. 261-275). Extraído de: http://www.redalyc.org/articulo.oa?id=39600514, consulta: 14/09/19

López, María. (2005). Capacitación. Conciencia Tecnológica, No. 27-30, Extraído de: http://www.redalyc.org/articulo.oa?id=94403013, consulta: 17/10/19

Majad, Musa. (2016). Gestión del talento humano en organizaciones educativas. Revista de Investigación. Vol.40, No.88, (Pp. 148-165). Extraído de: http://ve.scielo.org/pdf/ri/v40n88/art08.pdf, consulta: 09/08/19

Maya, Esther. (2014). Métodos y Técnicas de Investigación (primera edición electrónica). México. 
https://arquitectura.unam.mx/uploads/8/1/1/0/8110907/metodos_y_tecnicas.pdf, consulta: 29/08/19

Mejía, Armando., Bravo, Mario., y Montoya, Arturo. (2013). El factor del talento humano en las organizaciones. Ingeniería Industrial, Vol. XXXIV, No. 1, (Pp. 2-11). Extraído de: http://www.redalyc.org/articulo.oa?id=360433593002, consulta: 05/07/19

Osorio, Jaime., Navarrete, José., y Martínez, Carlos. (2009). Definición de un modelo de gestión para un banco de talentos empresariales en Pereira. Scientia Et Technica, vol. XV, No. 42, (Pp. 160-165). Extraído de: http://www.redalyc. org/articulo.oa?id=84916714030, consulta: 16/07/19

Restrepo, luz., Ladino, Ana., y Orozco, Diana. (2008). Modelo de reclutamiento y selección de Talento Humano por competencias para niveles directivo de la organización. Scientia Et Technica, Vol. XIV, No. 39, (Pp. 286-291). Extraído de: http://www.redalyc.org/articulo.oa?id=84920503051, consultado: $17 / 08 / 19$

Restrepo, Luz., y Ravagli, Ximena (2009). Modelo organizacional tendiente a potencializar el Talento Humano y mejorar su productividad. Scientia Et Technica, Vol. XV, No. 41, (Pp. 147-152). Extraído de: http://www.redalyc.org/articulo.oa?id=84916680026, consulta: $12 / 09 / 19$

Robbins, Stephen. (1998). Administración. Pearson Educación. México.

Robbins, Stephen., y Coulter, Mary. (2010). Administración (décima edición). Pearson. México. Extraído de: http://www.ru.edu.uy/wpcontent/uploads/2018/05/Administraci\%C3\%B3n-Robbins.pdf, consulta: 01/09/19

Rodríguez, Raúl., y Rosenstiehl, José (2018). Gestión de Operaciones y Talento Humano: Un modelo de elección discreta. Revista de la Facultad de Ciencias Económicas y Administrativas. Universidad de Nariño. Vol. XIX No. 2, (Pp92-112). Extraído en: http://dx.doi.org/10.22267/rtend.181902.99, consulta: 24/07/19

Salgado, Jesús y Cabal, Ángel. (2011). Evaluación del Desempeño en la Administración Pública del Principado de Asturias: Análisis de las Propiedades Psicométricas.

Revista de Psicología del Trabajo y de las Organizaciones. Vol. 27, No.2, (Pp.7591). Extraído de: https://doi.org/10.5093/tr2011v27n2a1, consulta: 25/08/19

Sapién, Alma., Piñón, Laura y Gutiérrez, María. (2014). Capacitación en la empresa mexicana: un estudio de formación en el trabajo Civilizar. Ciencias Sociales y Humanas, Vol. 14, No. 27, (Pp. 123-134). Extraído de: http://www.redalyc.org/articulo.oa?id=100235716007, consulta: 12/09/19

Suárez, Belkys. (2011). Posmodernidad: Dilemas de la gestión del talento humano en el ámbito empresarial. Observatorio Laboral Revista Venezolana, Vol. 4, No. 7, (Pp. 91-102). Extraído de: http://www.redalyc.org/articulo.oa?id=219022147006, consulta: 06/08/19 Tejada, Alonso. (2003). Los modelos actuales de gestión en las organizaciones. gestión del talento, gestión del conocimiento y gestión por competencias. Psicología desde el Caribe, núm. 12, (Pp. 115-133). Extraído de: http://www.redalyc.org/articulo.oa?id=21301208, consulta: 22/08/19 